TRANSACTIONS OF THE

AMERICAN MATHEMATICAL SOCIETY

Volume 352, Number 8, Pages 3495-3521

S 0002-9947(00)02569-1

Article electronically published on April 13, 2000

\title{
A WALL-CROSSING FORMULA FOR THE SIGNATURE OF SYMPLECTIC QUOTIENTS
}

\author{
DAVID S. METZLER
}

\begin{abstract}
We use symplectic cobordism, and the localization result of Ginzburg, Guillemin, and Karshon to find a wall-crossing formula for the signature of regular symplectic quotients of Hamiltonian torus actions. The formula is recursive, depending ultimately on fixed point data. In the case of a circle action, we obtain a formula for the signature of singular quotients as well. We also show how formulas for the Poincaré polynomial and the Euler characteristic (equivalent to those of Kirwan can be expressed in the same recursive manner.
\end{abstract}

\section{INTRODUCTION}

Since the original convexity theorems of Atiyah Ati82 and Guillemin-Sternberg GS82 on the image of the moment map associated to a Hamiltonian torus action, it has become clear that the moment polytope is a rich source of combinatorial invariants of these actions (see [Gui94]). In particular, traditional topological invariants of the various reduced spaces associated to the action turn out to have formulas that involve fixed point data combined with combinatorial data from the moment polytope. We will simply use the word "combinatorial" to refer to formulas of this type. In this paper we focus primarily on the signature, and find formulas for the signature of reduced spaces. In the circle case (Section 2) we are able to find formulas both for regular and singular reductions, thanks to recent work of Lerman and Tolman. In the torus case (Section 4) we find a recursive formula for the signatures of various "subreductions," including all regular reductions.

Our fundamental object of study is a Hamiltonian $T$-space $M$, with symplectic form $\omega$ and moment map $\phi: M \rightarrow \mathfrak{t}^{*}$. We denote such a Hamiltonian $T$-space by $(M, \omega, \phi)$. We allow our spaces to be orbifolds as well as manifolds. We will usually restrict ourselves to compact spaces.

Given a point $a \in \mathfrak{t}^{*}$, the symplectic reduction, or symplectic quotient of $M$ at $a$, is defined to be

$$
M_{a}=\phi^{-1}(a) / T,
$$

sometimes denoted $(M / / T)_{a}$ (when we need to explicitly include $T$ ). Recall that if $a$ is a regular value of $\phi, M_{a}$ is a smooth symplectic orbifold, and that the symplectic form $\omega_{a}$ on $M_{a}$ is defined by the requirement that

$$
\pi^{*} \omega_{a}=i^{*} \omega
$$

Received by the editors September 20, 1998.

2000 Mathematics Subject Classification. Primary 53D20; Secondary 57R85.

Key words and phrases. Symplectic geometry, Hamiltonian action, equivariant cobordism.

(C) 2000 American Mathematical Society 
where $\pi: \phi^{-1}(a) \rightarrow M_{a}$ and $i: \phi^{-1}(a) \rightarrow M$ are the projection and inclusion maps, respectively.

Let $\Delta_{\text {reg }}$ be the set of regular values of the moment map. We will see in Section 3 that this is a finite union of open convex polytopes (see also GS82]), which we call chambers. When computing topological invariants of reduced spaces, the essential fact is

Proposition $1.1([\overline{\mathrm{DH} 82}])$. Let $(M, \omega, \phi)$ be a compact, connected Hamiltonian $T$ space. Let $a, b \in \Delta_{\text {reg }}$ lie in the same chamber $P$. Then the two reduced spaces $M_{a}$ and $M_{b}$ are diffeomorphic.

Hence any topological invariant of the reduced spaces $M_{a}\left(a \in \Delta_{r e g}\right)$ will be a function only of the chamber that $a$ is in, making it essentially combinatorial.

In Section 2 we use the cobordism results of Guillemin, Ginzburg and Karshon GGK96 to derive a formula for the signature of a regular reduced space in the case of a circle action, in terms of fixed point data. This formula is best expressed as a "wall-crossing" formula. This form is quite simple, and generalizes best to the torus case. A wall-crossing formula expresses the change in an invariant of the reduced space when one crosses a singular value of the moment map. Wall-crossing formulas for invariants of reduced spaces have a long history, including the formula in [GS89] for the change in the cohomology class [ $\omega_{\text {red] }}$, and the formula in GLS96] for the change in the Duistermaat-Heckman polynomial.

In Section 2.4 we analyze the case of singular reduction by a circle action, using recent results of Lerman and Tolman $[\mathrm{LT}]$. Again we obtain a simple formula in terms of fixed point data.

Let us make precise what we mean by "fixed point data." If $M$ is a compact $T$ space, the fixed point set $M^{T}$ has finitely many connected components $F_{1}, \ldots, F_{m}$. Each $F_{r}$ is a compact connected manifold. If $M$ is Hamiltonian, it has associated to it a natural isotopy class of $T$-invariant almost complex structures [MS95]. Hence the normal bundle $N_{r}$ to each $F_{r}$ has a natural isotopy class of complex structures, which allows us to define the weights of the action of $T$ on the normal bundle. Call these weights $\alpha_{r, k} \in \mathfrak{t}^{*}, k=1, \ldots, \operatorname{rank}\left(N_{r}\right) / 2$. It is easy to see that the moment map $\phi$ must be constant on each $F_{r}$, so each component $F_{r}$ defines a point in $\mathfrak{t}^{*}$, $\phi_{r}=\phi\left(F_{r}\right)$. The manifolds $F_{r}$, together with the weights $\alpha_{r, k}$ and the points $\phi_{r}$, comprise the fixed point data that our formulas ultimately depend on.

To investigate the torus case, we first introduce a variant of the $X$-ray of Tolman Tol98], and describe it in Section 3 This is a package of data that includes the fixed point data, but also takes into account the fixed point sets of subtori $H \subset T$. Using this language in Section 4, we find a recursive generalization of the wall-crossing formula obtained in the circle case, where the data involved in crossing a wall of dimension $d$ is obtained by a wall-crossing procedure in dimension $d-1$. In some cases this reduces to a formula which is as simple as the one in the circle case.

We conclude by deriving similar recursive formulas for the Poincaré polynomial (and hence the Euler characteristic). Kirwan [Kir84] gives explicit formulas for these quantities, but our formulas are perhaps more geometrical and easier to calculate than hers.

This paper is largely derived from the author's Ph.D. thesis [Met97, supervised by Victor Guillemin. The author would also like to gratefully acknowledge the support and advice of Eugene Lerman, Sue Tolman, and Yael Karshon. Reyer 
Sjamaar provided valuable comments. The author is especially grateful to the referee for a careful perusal of the paper and many comments and emendations.

\section{Circle actions}

Since the signature is a cobordism invariant, it is natural to use cobordism arguments to try to calculate it. Guillemin, Ginzburg, and Karshon GGK96 have introduced a notion of cobordism of spaces with Hamiltonian group actions which is perfectly suited to our task. It has two main features: First, a cobordism of Hamiltonian spaces induces cobordisms of their reduced spaces. Second, there is a localization theorem, which says that every space is cobordant to a sum of local models, determined by the fixed point data. Further, it was noticed by Karshon Kar98 that the "moment map" that appears in their definition of cobordism need not actually come from a symplectic form, but must only satisfy some simple axioms. This will prove useful in investigating the signature of singular reductions.

Note. as has become standard in studying symplectic reduction, we will deal everywhere with orbifolds, since they arise naturally from reduction anyway. For our purposes this introduces essentially no difficulties. See [LT97] for foundational background on Hamiltonian actions on orbifolds.

2.1. Abstract moment maps and cobordism. Let $M$ be an orbifold with an action of a torus $T$. Given a subgroup $H \subset T$, let $\pi_{H}: \mathfrak{t}^{*} \rightarrow \mathfrak{h}^{*}$ denote the natural projection map, and let $M^{H}$ be the set of fixed points of $H$.

Definition 2.1. An abstract moment map for $M$ is a $T$-invariant map $\phi: M \rightarrow$ $\mathfrak{t}^{*}$ such that, for every subgroup $H \subset T$, the composed map

$$
\left.\pi_{H} \circ \phi\right|_{M^{H}}: M^{H} \rightarrow \mathfrak{t}^{*} \rightarrow \mathfrak{h}^{*}
$$

is locally constant.

It is easy to show that if $(M, \omega, \phi)$ is a Hamiltonian $T$-space, then the moment map $\phi$ is an abstract moment map. However, abstract moment maps are much looser objects (for example, the zero map is trivially an abstract moment map) and they do not depend on a symplectic form for their definition. However, the notion of reduction still makes sense: it is easy to see that if $a \in \mathfrak{t}^{*}$ is a regular value of $\phi$, then the action of $T$ on $\phi^{-1}(a)$ is locally free, and hence $\phi^{-1}(a) / T$ is an orbifold, denoted by $M_{a}$.

We will need to be careful about orientations of reduced spaces. In the symplectic case, the reduced space is clearly oriented, being symplectic. However, even in the case of an abstract moment map, the reduced space has a canonical orientation, as long as $M$ is itself oriented. We have short exact sequences

$$
\begin{aligned}
& 0 \longrightarrow \mathfrak{t} \stackrel{\iota}{\longrightarrow} T_{p}\left(\phi^{-1}(a)\right) \rightarrow T_{[p]}\left(M_{a}\right)>0 \\
& 0 \rightarrow T_{p}\left(\phi^{-1}(a)\right) \longrightarrow T_{p} M \stackrel{d \phi}{\longrightarrow} \mathfrak{t}^{*} \longrightarrow 0
\end{aligned}
$$

where $\iota$ denotes the infinitesimal action of the torus. Hence if we choose an orientation for $\mathfrak{t}$ (and hence for $\mathfrak{t}^{*}$ ) we obtain orientations on $\phi^{-1}(a)$ and $M_{a}$. If we reverse the orientation of $\mathfrak{t}$, the orientation on $\mathfrak{t}^{*}$ also reverses, and hence the orientation on $M_{a}$ is unchanged, so it is actually well-defined independent of the orientation of t. 
We can now introduce the notion of cobordism that we need. Let $\left(M_{1}, \phi_{1}\right)$ and $\left(M_{2}, \phi_{2}\right)$ be two oriented $T$-spaces with proper abstract moment maps.

Definition 2.2. A cobordism between $\left(M_{1}, \phi_{1}\right)$ and $\left(M_{2}, \phi_{2}\right)$ is an oriented orbifold $W$ and a proper abstract moment map $\psi: W \rightarrow \mathfrak{t}^{*}$, such that $\partial W=M_{1} \amalg \bar{M}_{2}$ and $\left.\psi\right|_{M_{i}}=\phi_{i}$.

The overbar indicates reversed orientation. The key to this definition is the word "proper"; the orbifold $W$ need not be compact, but requiring the moment map to be proper ensures that the concept is non-trivial.

In the case where $(M, \phi)$ arises from an honest Hamiltonian $T$-space, we require the cobording manifold $W$ to carry a presymplectic form (i.e. a closed 2-form) which restricts to the given forms on the boundary components, and we require the abstract moment map $\psi$ to be an ordinary moment map for this presymplectic form. In this case we will say that the spaces are cobordant as Hamiltonian $T$-spaces.

This notion of cobordism respects reduction:

Proposition 2.3 ([Kar98] $)$. Let $\left(M_{1}, \phi_{1}\right)$ and $\left(M_{2}, \phi_{2}\right)$ be cobordant as oriented $T$-spaces with abstract moment maps. Let $a \in \mathfrak{t}^{*}$ be a regular value of $\phi_{1}$ and $\phi_{2}$. Then the reduced spaces $\left(M_{1}\right)_{a}$ and $\left(M_{2}\right)_{a}$, with the orientations as defined above, are cobordant (as compact oriented orbifold:11).

The crucial result from GGK96] and [Kar98] is the localization theorem. This can be seen as a geometric antecedent to the theorems of Duistermaat and Heckman [DH82] and of Jeffrey and Kirwan [JK95.

From now on we will restrict to the case where $T=S^{1}$, since this is all we need for the signature formula. This makes the statements simpler, although the essential picture is the same for higher rank tori.

We need some preliminaries about orientations. Let $M$ be an oriented, compact $T$-space with abstract moment map $\phi$. Denote the components of the fixed point set by $F_{1}, \ldots, F_{m}$. Fix once and for all an identification of $\mathfrak{t}$ with $\mathbb{R}$ (a "polarization" of $\mathfrak{t})$. Given a fixed point component $F_{r}$, consider the real weights of the action of $T$ on $N_{r}$. These are nonzero integers defined only up to sign, and we will denote them $\pm \alpha_{r, k}, k=1, \ldots, q_{r}$, where $q_{r}=\operatorname{rank}\left(N_{r}\right) / 2$. By $\alpha_{r, k}^{\#}$ we will mean the weight with the positive choice of sign; we will refer to these as polarized weights.

Fix temporarily an orientation of $F_{r}$. This determines an orientation of $N_{r}$, and hence makes the Pfaffian, Pf, well-defined on the fibers of $N_{r}$. Let $\xi \in \mathfrak{t}$ be positive, and let $\xi_{p}$ be the isotropy action of $\xi$ on the fiber of $N_{r}$ over some $p \in F_{r}$. Let

$$
\varepsilon_{r}=\operatorname{sgn} \operatorname{Pf}\left(\xi_{p}\right)
$$

which is clearly independent of the choice of $p$.

If we reverse the orientation of $F_{r}$, it is clear that the sign of $\varepsilon_{r}$ reverses as well. Hence there is a unique orientation of $F_{r}$ which makes $\varepsilon_{r}$ positive. Denote by $F_{r}^{\#}$ the fixed point component with this choice of orientation, which we will refer to as the polarized orientation. (If $F_{r}$ is a single point, this is just a formal sign attached to $F_{r}$.) We will also refer to the corresponding orientation on the bundle as the polarized orientation.

\footnotetext{
${ }^{1}$ Note that even if $M_{1}$ and $M_{2}$ are manifolds, we allow them to be cobordant by an orbifold.
} 
We note that in the Hamiltonian case, this orientation does not necessarily agree with the symplectic orientation on $F_{r}$. To be precise, let $M$ be a Hamiltonian $T$ space. Use a compatible complex structure on the normal bundle $N_{r}$ to identify complex weights $\alpha_{r, k}$. These are honest integers, not just up to sign. Let $\sigma_{r}$ be the number of negative complex weights. If we use the symplectic orientation on $F_{r}$, it is not hard to see that the sign of the Pfaffian of the action is $\varepsilon_{r}=(-1)^{\sigma_{r}}$. Hence the symplectic orientation differs from the polarized orientation by $(-1)^{\sigma_{r}}$. In particular, when the component $F_{r}$ is a single point $p$, the formal sign we attach to it is exactly $(-1)^{\sigma_{r}}$. This will be crucial in getting the correct signs in the signature formula (2.5).

The localization theorem says roughly that the whole space $M$ is cobordant to the disjoint union of the normal bundles of the fixed point components, with appropriately defined moment maps.

Fix an $N_{r}$, and equip it with an equivariant inner product. The bundle splits into isotypic subbundles corresponding to distinct real weights $\alpha_{r, k}$. (The rank of the subbundle corresponding to a certain weight $\beta$ is twice the number of values of $k$ with $\alpha_{r, k}=\beta$.) Denote the corresponding splitting of any $v \in N_{r}$ by $v=\sum v_{k}$. Define a map on the bundle by

$$
\psi_{r}(v)=\phi\left(F_{r}\right)+\sum_{k=1}^{q_{r}}\left\|v_{k}\right\|^{2} \alpha_{r, k}^{\#} .
$$

This is a proper moment map for the $T$-space $N_{r}$.

We are now ready to state the localization theorem.

Theorem 2.4 ([Kar98]). Let $T=S^{1}$. Let $M$ be a compact $T$-space with abstract moment map $\phi$. Fix a polarization for T. Define the polarized weights $\alpha_{r, k}^{\#}$ and the maps $\psi_{r}$ as above. Then $M$ is cobordant, as a $T$-space with abstract moment map, to the disjoint union of the normal bundles:

$$
(M, \phi) \sim \coprod_{r}\left(N_{r}, \psi_{r}\right)
$$

(Note: with the appropriate definition of a polarization and of the moment maps on the normal bundles, essentially the same statement applies in the higher-rank torus case.)

2.2. Cobordism and reduction. It remains to state what happens under reduction. The localization theorem says that the original space is cobordant to a sum of local models, so any reduction will be cobordant to a sum of reduced local models. In the circle case it is particularly easy to see what these reduced local models are. We begin by analyzing the reduction of a single vector space.

Let $V$ be a real orthogonal $T$-representation with real weights

$$
\pm \alpha_{1}, \ldots, \pm \alpha_{q} \neq 0
$$

Give $V$ the polarized orientation (so that the Pfaffian of the action of a positive generator of $\mathfrak{t}$ is positive, as discussed above). Fix an element $b \in \mathfrak{t}^{*}$. Equip $V$ with the map $\psi_{\alpha, b}: V \rightarrow \mathfrak{t}^{*}$ given by

$$
\psi_{\alpha, b}(v)=b+\sum_{k=1}^{q}\left\|v_{k}\right\|^{2} \alpha_{k}^{\#}
$$


Denote this oriented $T$-space with abstract moment map by $V(\alpha, b)$. As a vector space it is just $\mathbb{C}^{n}$.

Given such a local model $V(\alpha, b)$, and a point $a \in \mathfrak{t}^{*}, a \neq b$, there are two possibilities for the reduction of $V$ at $a$. If $a<b$, then the reduction is empty, since the image of $\psi_{\alpha, b}$ lies to the right of $b$. If $a>b$, then the reduction is

$$
\begin{aligned}
V(\alpha, b)_{a} & =\left(\psi_{\alpha, b}\right)^{-1}(a) / S^{1} \\
& =\left\{\left.v \in V\left|b+\frac{1}{2} \sum_{k=1}^{q}\right| v_{k}\right|^{2} \alpha_{k}^{\#}=a\right\} / S^{1}
\end{aligned}
$$

which is a twisted complex projective space of dimension $2 q-2$. Denote the (possibly empty) reduced space by $X(\alpha, a, b)$. This is generally an orbifold, but (when it is nonempty) its rational cohomology ring is isomorphic to that of the ordinary projective space $\mathbb{C P}^{n-1}$. Further, it is not hard to see that the orientation that $X(\alpha, a, b)$ inherits as a reduced space is the same as the complex orientation it has under the identification with a twisted $\mathbb{C P}^{n-1}$, because of the condition we put on the orientation of $V$.

The case of a vector bundle is not much more complicated. Let $V$ be a real, orientable orthogonal $T$-bundle over a trivial $T$-space $F$, with real weights $\pm \alpha_{1}, \ldots$, $\pm \alpha_{q} \neq 0$. Again give $E$ the polarized orientation. Fix an element $b \in \mathfrak{t}^{*}$. Equip $E$ with the map $\psi_{\alpha, b}: E \rightarrow \mathfrak{t}^{*}$ given by

$$
\psi_{\alpha, b}(v)=b+\sum_{k=1}^{q}\left\|v_{k}\right\|^{2} \alpha_{k}^{\#} .
$$

This is an oriented $T$-space with abstract moment map, whose fibers are copies of $V(\alpha, b)$.

Since $E$ fibers equivariantly over the trivial $T$-space $F$, so will the reduction. Since the fibers are simply copies of $V(\alpha, b)$, the fibers of the reduced space $E_{a}$ will be copies of $X(\alpha, a, b)$. Hence the only spaces we have to deal with are bundles whose fibers are twisted complex projective spaces.

Note also that $E_{a}$ is an associated bundle of the oriented orthonormal frame bundle of $E$. If the $T$-action on the fibers of $E$ commutes with the action of the structure group, the structure group acts naturally on the reduction by $T$. In particular, the structure group of the reduced space as a bundle over $F$ is compact and connected.

Combining these local results with the localization theorem, and the fact that cobordism respects reduction, we have

Theorem 2.5. Let $T=S^{1}$, and choose a fixed polarization of $T$. Let $M$ be a compact oriented $T$-space with abstract moment map $\phi$. Give each fixed point component $F_{r}$ its polarized orientation $F_{r}^{\#}$. Let $a \in \mathfrak{t}^{*}$ be a regular value of $\phi$. Then there is an oriented orbifold cobordism

$$
M_{a} \sim \coprod_{r}\left(N_{r}^{\#}\right)_{a}
$$

where each $\left(N_{r}^{\#}\right)_{a}$ is a fiber bundle over $F_{r}^{\#}$ with fiber $X_{r}=X\left(\alpha_{r}, a, \phi\left(F_{r}\right)\right)$, and with compact connected structure group. The fibers $X_{r}$ are orientation-preserving diffeomorphic to twisted complex projective spaces. 
If $M$ is Hamiltonian, then the polarized orientation $F_{r}^{\#}$ differs from the symplectic orientation of $F_{r}$ by $(-1)^{\sigma_{r}}$, where $\sigma_{r}$ is the number of negative weights of the isotropy action at $F_{r}$.

2.3. The signature formula in the circle case. Among the topological invariants that are preserved by cobordism, there is one that is singled out by being multiplicative for any fiber bundle with oriented fiber and compact connected structure group: the signature. We recall

Definition 2.6. Given a connected, compact, oriented $4 k$-dimensional rational homology manifold $M$, the signature $\operatorname{Sign}(M)$ is defined to be the signature of the symmetric bilinear pairing $H^{2 k}(M, \mathbb{Q}) \otimes H^{2 k}(M, \mathbb{Q}) \rightarrow H^{4 k}(M, \mathbb{Q}) \cong \mathbb{Q}$ given by the cup product. If $\operatorname{dim} M$ is not divisible by 4 , we define $\operatorname{Sign}(M)=0$.

Note that any orbifold is a rational homology manifold [Ful93], so it has a welldefined signature.

The signature is of course an important and natural invariant of a manifold (or orbifold). Its special significance to this work is expressed by the following three results.

Theorem 2.7. The signature is an invariant of oriented orbifold cobordism.

Proof. This was proved for manifolds by Thom (see Hirzebruch [Hir66]). The proof relies only on Poincaré duality, hence it works for rational homology manifolds, hence for orbifolds.

The second result concerns the multiplicativity of the signature, and is a slight modification of a result of Chern, Hirzebruch, and Serre [CHS57]:

Theorem 2.8. Let $E \rightarrow B$ be a fiber bundle with fiber $F$ such that

1. E, B, $F$ are compact connected oriented orbifolds;

2. the structure group of $E$ is compact and connected.

If $E, B, F$ are oriented coherently, then

$$
\operatorname{Sign}(E)=\operatorname{Sign}(B) \operatorname{Sign}(F) .
$$

Proof. Chern, Hirzebruch and Serre prove that the conclusion follows from condition (11), with "manifold" in place of orbifold, and the following condition:

$2^{\prime}$. The fundamental group $\pi_{1}(B)$ acts trivially on $H^{*}(F)$.

As above, their proof uses only Poincaré duality (and the spectral sequence of a fibration), and hence applies equally well to rational homology manifolds, such as orbifolds. Also, condition (2) implies $\left(2^{\prime}\right)$. For, the action of $\pi_{1}(B)$ factors through the structure group; and the action of a compact connected group on cohomology is trivial. Our version of the theorem then follows.

To emphasize the special role of the signature, we briefly mention

Theorem 2.9 (Borel, Hirzebruch; see [HBJ92]). The signature is the only rational invariant of oriented cobordism that is multiplicative in the sense of Theorem [2.8 and takes the value 1 on $\mathbb{C P}^{2}$. 
Theorems 2.7 and 2.8 together with Theorem 2.5, immediately give the formula for the signature of the reduction by a circle action:

$$
\begin{aligned}
\operatorname{Sign}\left(M_{a}\right) & =\sum_{r} \operatorname{Sign}\left(F_{r}^{\#}\right) \operatorname{Sign}\left(X_{r}\right) \\
& =\sum_{r}(-1)^{\sigma_{r}} \operatorname{Sign}\left(F_{r}\right) \operatorname{Sign}\left(X_{r}\right)
\end{aligned}
$$

where the second equation applies to the Hamiltonian case, in which $F_{r}$ is given its symplectic orientation.

In the circle case the computation of $\operatorname{Sign}\left(X_{r}\right)$ is quite simple, since $X_{r}$ is either empty, or a twisted complex projective space of complex dimension $q_{r}-1$, where $q_{r}$ is the half-rank of the normal bundle $N_{r}$. The signature of a twisted complex projective space is just 1 when its complex dimension is even, 0 when it is odd.

To be precise, fix a polarization of $T$. Let $F_{1}, \ldots, F_{m}$ be the fixed point components, and let $\phi_{r}=\phi\left(F_{r}\right)$. In the Hamiltonian case, let $f_{r}$ and $b_{r}$ be the number of positive and negative weights, respectively, of $F_{r}\left(\right.$ so $\left.f_{r}+b_{r}=q_{r}\right)$. Then the above formula becomes the following:

Theorem 2.10. The signature of a regular reduction of a space with circle action and abstract moment map is given by

$$
\begin{aligned}
\operatorname{Sign}\left(M_{a}\right) & =\sum_{r: \phi_{r}<a, q_{r} \text { odd }} \operatorname{Sign}\left(F_{r}^{\#}\right) \\
& =\sum_{r: \phi_{r}<a, q_{r} \text { odd }}(-1)^{b_{r}} \operatorname{Sign}\left(F_{r}\right),
\end{aligned}
$$

where the latter equation applies only to the Hamiltonian case, and $F_{r}$ is given its symplectic orientation.

This can be restated as a wall-crossing formula, which will be more convenient later.

Fix a singular value $c \in \mathfrak{t}^{*}$ of the moment map. Let $F_{1}, \ldots, F_{m}$ be the fixed point components with $\phi\left(F_{r}\right)=c$. Pick $a_{1}<c<a_{2}$ such that there are no other singular values in the interval $\left[a_{1}, a_{2}\right]$. (See Figure 1)

Denote the half-rank of the normal bundle $N_{r}$ by $q_{r}$. Then

$$
\operatorname{Sign}\left(M_{a_{2}}\right)-\operatorname{Sign}\left(M_{a_{1}}\right)=\sum_{r=1 \ldots m, q_{r} \text { odd }}^{m} \operatorname{Sign}\left(F_{r}^{\#}\right) .
$$

In the Hamiltonian case, we have a slightly more explicit formula:

Define the function $w_{S}: \mathbb{N} \times \mathbb{N} \rightarrow \mathbb{Z}$ by

$$
w_{S}(f, b):=\left\{\begin{array}{cl}
(-1)^{b} & \text { if } f+b \text { is odd } \\
0 & \text { if } f+b \text { is even. }
\end{array}\right.
$$

Then

$$
\operatorname{Sign}\left(M_{a_{2}}\right)-\operatorname{Sign}\left(M_{a_{1}}\right)=\sum_{r=1}^{m} w_{S}\left(f_{r}, b_{r}\right) \operatorname{Sign}\left(F_{r}\right) .
$$

Here $F_{r}$ has its symplectic orientation.

The idea of "wall-crossing," and generalizations of (2.8) will be central to what follows. 


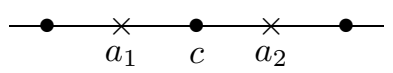

Figure 1. Wall crossing in the circle case

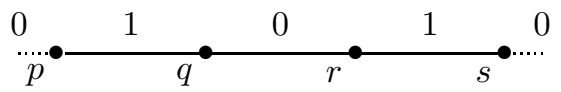

FiguRe 2. Wall crossing example: $\mathbb{C P}^{3}$ with circle action

Example. We first describe a whole family of simple examples of Hamiltonian torus actions. Let $M=\mathbb{C P}^{n}$. This has a natural action of $T^{n+1}$ inherited from the standard linear action of $T^{n+1}$ on $\mathbb{C}^{n+1}$

$$
\left(\lambda_{1}, \ldots, \lambda_{n+1}\right) \cdot\left(z_{1}, \ldots, z_{n+1}\right)=\left(\lambda_{1} z_{1}, \ldots, \lambda_{n+1} z_{n+1}\right) .
$$

(This action is not effective, since the diagonal circle acts trivially on $\mathbb{C P}^{n}$.) This action is easily seen to be Hamiltonian, with moment map

$$
\phi\left(\left[z_{1}, \ldots, z_{n+1}\right]\right)=\frac{1}{\|z\|} \sum_{k=1}^{n+1}\left|z_{k}\right|^{2} e_{k},
$$

whose image is exactly the standard $n$-simplex in $\mathbb{R}^{n+1}$,

$$
\Delta_{n}=\left\{a_{1}, \ldots, a_{n+1} \mid \sum a_{k}=1\right\} .
$$

Choose a subtorus $T \subset T^{n+1}$ of rank $d$, which does not contain the diagonal circle. Then $M$ is an effective Hamiltonian $T$-space, and the moment map is just $\phi$ composed with the projection $\mathbb{R}^{n+1} \rightarrow \mathfrak{t}^{*}$. The moment polytope is the image of the standard $n$-simplex under this projection. If $T$ is generic, its fixed points are the same as those of $T^{n+1}$, which are clearly the $n+1$ isolated points $\left[z_{1}, \ldots, z_{n+1}\right]$ with $z_{j}=\delta_{k}^{j}$, for $k=1, \ldots, n+1$.

For now, choose $n=3, d=1$, so we get a generic Hamiltonian circle action on $\mathbb{C P}^{3}$, with 4 isolated fixed points. The moment polytope is shown in Figure 2, with the images of the fixed points shown as $p, q, r, s$. Since the complex dimension is 3 , each fixed point has 3 weights associated to it. It is not hard to see by looking at the action that at $p$, all three weights point to the right; at $s$, all three point left; at $q$, one points left, two point right; and at $r$, two point left and one points right.

Since all fixed points are isolated, the wall-crossing rule (2.8) gives the signatures shown in the figure. These agree with a direct calculation of the reduced spaces, which easily shows that the reductions in the outer two chambers are (twisted) $\mathbb{C P}^{2}$, which has signature 1 , and the reduction in the inner chamber has the rational cohomology of $\mathbb{C P}^{1} \times \mathbb{C P}^{1}$, which has signature 0 . 
2.4. Singular reduction. Recently Lerman and Tolman $[\mathrm{LT}]$ have found a beautiful way to analyze the rational intersection cohomology ring of singular reductions for Hamiltonian circle actions. 2 We can use a piece of their result to easily extend the above formula to the singular case. The key is that Theorem 2.10 applies to any abstract moment map.

Given a singular value $c$ of the moment map, let $\mathcal{F}_{c}=\left\{F_{1}, \ldots, F_{m}\right\}$ be the set of fixed point components with $\phi\left(F_{k}\right)=c$, as above. Divide $\mathcal{F}_{c}$ into two disjoint sets

$$
\begin{aligned}
& \mathcal{F}_{c}^{+}=\left\{F_{k} \mid b_{k}<f_{k}\right\}, \\
& \mathcal{F}_{c}^{-}=\left\{F_{k} \mid b_{k} \geq f_{k}\right\} .
\end{aligned}
$$

Lerman and Tolman show that by deforming the moment map $\phi$ to a new map $\tilde{\phi}$, which is now only an abstract moment map, they can find a "small resolution" of the singularities of the singular reduction $\phi^{-1}(c) / S^{1}$. Precisely,

Theorem $2.11\left([\mathrm{LT})\right.$. Let $(M, \omega, \phi)$ be a Hamiltonian $S^{1}$-space. Let $c$ be a singular value of $\phi$, and define $F_{1}, \ldots, F_{m}, \mathcal{F}_{c}^{ \pm}$as above. Then there exist $a, b \in \mathbb{R}$, $a<c<b$, and an abstract moment map $\tilde{\phi}$, such that

1. $a, b$ are regular values of both $\phi$ and $\tilde{\phi}$;

2. there are no fixed points in $\phi^{-1}([a, b])$ besides those in $\mathcal{F}_{c}$;

3. $\tilde{\phi}$ and $\phi$ are equal on $M \backslash \phi^{-1}([a, b])$;

4. $c$ is a regular value of $\tilde{\phi}$;

5. $a<\tilde{\phi}\left(F_{k}\right)<c$ for all $F_{k} \in \mathcal{F}_{c}^{-}$and $c<\tilde{\phi}\left(F_{k}\right)<b$ for all $F_{k} \in \mathcal{F}_{c}^{+}$;

6. $\tilde{M}_{c}:=\tilde{\phi}^{-1}(c) / S^{1}$ is a small resolution of $M_{c}=\phi^{-1}(c) / S^{1}$.

7. There is a pairing-preserving isomorphism between the (middle perversity) intersection cohomology of $M_{c}$ and the ordinary cohomology of $\tilde{M}_{c}$.

We can now apply our formulas to the new abstract moment map $\tilde{\phi}$ to get a formula for the singular reduction. We simply compare $\tilde{M}_{c}$ to $M_{a}$ (or to $M_{b}$ ).

The wall-crossing formula (2.8) applied to $\tilde{\phi}$ gives

$$
\operatorname{Sign}\left(\tilde{M}_{c}\right)=\operatorname{Sign}\left(\tilde{M}_{a}\right)+\sum_{F_{r} \in \mathcal{F}_{c}^{-}, \quad q_{r} \text { odd }} \operatorname{Sign}\left(F_{r}^{\#}\right) .
$$

One gets a similar formula involving $\mathcal{F}_{c}^{+}$by comparing to $\tilde{M}_{b}$.

Note that since the $F_{r}$ are actually fixed point components of the original Hamiltonian action, they are symplectic, and the normal bundles do have complex structures. So we can restate this formula in terms of the symplectic orientation of $F_{r}$ and the number of negative weights, just as in the honest Hamiltonian case

$$
\operatorname{Sign}\left(\tilde{M}_{c}\right)=\operatorname{Sign}\left(\tilde{M}_{a}\right)+\sum_{F_{r} \in \mathcal{F}_{c}^{-}} w_{S}\left(f_{r}, b_{r}\right) \operatorname{Sign}\left(F_{r}\right) .
$$

Since $\tilde{M}_{c}$ is a small resolution of $M_{c}$, their signatures are equal (where the signature of the singular space $M_{c}$ is understood to be taken in intersection cohomology). Also, since $\tilde{\phi}$ and $\phi$ only differ near the level $c$, we have $\tilde{M}_{a}=M_{a}$, and $\tilde{M}_{b}=M_{b}$.

Hence we have proved

\footnotetext{
${ }^{2}$ See [Kir88] for background on intersection cohomology.
} 
Theorem 2.12. Let $(M, \omega, \phi)$ be a compact Hamiltonian $S^{1}$-space, and let $c$ be a singular value of the moment map. Define the sets $\mathcal{F}_{c}^{ \pm}$and the function $w_{S}$ as above. Choose $a, b \in \mathbb{R}, a<c<b$, such that there are no critical values of $\phi$ in the intervals $[a, c)$ and $(c, b]$. Then

$$
\begin{aligned}
\operatorname{Sign}\left(M_{c}\right) & =\operatorname{Sign}\left(M_{a}\right)+\sum_{F_{r} \in \mathcal{F}_{c}^{-}} w_{S}\left(b_{r}, f_{r}\right) \operatorname{Sign}\left(F_{r}\right) \\
& =\operatorname{Sign}\left(M_{b}\right)-\sum_{F_{r} \in \mathcal{F}_{c}^{+}} w_{S}\left(b_{r}, f_{r}\right) \operatorname{Sign}\left(F_{r}\right) .
\end{aligned}
$$

Note that the jump between the signatures of the regular reductions $\operatorname{Sign}\left(M_{a}\right)$ and $\operatorname{Sign}\left(M_{b}\right)$, which is equal to

$$
\sum_{F_{r} \in \mathcal{F}_{c}} w_{S}\left(b_{r}, f_{r}\right) \operatorname{Sign}\left(F_{r}\right)
$$

is simply divided into two steps, one between $\operatorname{Sign}\left(M_{a}\right)$ and $\operatorname{Sign}\left(M_{c}\right)$, the other between $\operatorname{Sign}\left(M_{c}\right)$ and $\operatorname{Sign}\left(M_{b}\right)$. Each fixed point component contributes to one step or the other depending on whether it has more weights up or down.

One may wonder why in the definitions of $\mathcal{F}_{c}^{ \pm}$we chose the $F_{r}$ with $b_{r}=f_{r}$ to lie in $\mathcal{F}_{c}^{-}$and not in $\mathcal{F}_{c}^{+}$. This is in fact an arbitrary choice; Lerman and Tolman's theorem is still true if we put these $F_{r}$ in $\mathcal{F}_{c}^{+}$instead. Precisely, making this choice gives a different small resolution $\tilde{M}_{c}^{\prime}$ of $M_{c}$, with a cohomology ring that is not isomorphic to the one resulting from our choice; however, the pairings are isomorphic. This makes sense in Theorem 2.12 because the fixed point components with $b_{r}=f_{r}$ do not contribute to the wall-crossing: when $b_{r}=f_{r}, b_{r}+f_{r}$ is even and $w_{S}\left(b_{r}, f_{r}\right)=0$. Hence it doesn't matter where we include them.

\section{The STRuCture of The MOMENT POLYTOPE}

To describe the formula for the signature in the torus case, we first need to review what is known about the structure of the moment polytope, and introduce some notation.

Let $T$ be a torus of rank $d$. Let $(M, \omega, \phi)$ be a compact Hamiltonian $T$-space of dimension $2 n$. For convenience, assume the action is effective. By the convexity theorem of Atiyah Ati82 and Guillemin and Sternberg GS82, the moment image $\Delta=\phi(M)$ is a convex polytope, and is in fact the convex hull of the image the fixed point set $M^{T}$. However, one can say a great deal more about the structure of $\Delta$. We will introduce a refinement of Tolman's notion of the $X$-ray of $M$ to provide the framework for later calculations.

Note. Our definition of X-ray will differ in one important respect from Tolman's. Tolman works with the stratification of $M$ by orbit type, whereas we will work with the infinitesimal orbit type stratification. This is equivalent to only considering the connected component of the identity in each stabilizer subgroup. This gives, in general, a strictly coarser stratification than the one which Tolman works with. By considering the full orbit-type stratification, Tolman obtains torsion information, in particular, the intrinsic stabilizers of singular points in the reduced spaces (when these spaces are orbifolds). However, we are primarily interested in rational invariants, which are insensitive to this information. We could refer to our X-rays as "X-rays modulo torsion." 
Recall from the structure theory of compact transformation groups (see e.g. Kaw91) that $M$ decomposes into a finite set of (infinitesimal) orbit-type strata. Given a connected subgroup $H \subset T$, with Lie algebra $\mathfrak{h}$, the orbit-type stratum $M_{H}$ is the set of points $p \in M$ such that the connected component of the isotropy subgroup $T_{p}$ is $H$, or equivalently, such that the infinitesimal isotropy $\mathfrak{t}_{p}=\mathfrak{h}$. Let $X_{1}, \ldots, X_{m}$ be the set of connected components of infinitesimal orbit-type strata, with corresponding connected isotropy subgroups $T_{1}, \ldots, T_{m}$. For each $X_{j}$ denote the closure by $F_{j}=\bar{X}_{j}$. We will abuse notation and refer to these as the strata of $M$. Let $\mathcal{F}=\left\{F_{1}, \ldots, F_{m}\right\}$ be the set of strata. Each $F_{j}$ is a connected component of the fixed point set $M^{T_{j}}$ of the subtorus $T_{j}$. Note that $T_{j}$ is the stabilizer of a generic point on $F_{j}$, but some points in $F_{j}$ will have a larger stabilizer. Denote $\operatorname{dim}\left(T_{j}\right)$ by $d_{j}$.

Each $F_{j}$ is a symplectic manifold in its own right, by the equivariant Darboux theorem GS84. In fact, the restriction of the moment map $\phi$ makes $F_{j}$ into a Hamiltonian $T$-space. However, the $T$-action on $F_{j}$ is clearly not effective. $F_{j}$ inherits an effective action of the quotient torus $T / T_{j}$. Since $T_{j}$ acts trivially, $\phi\left(F_{j}\right)$ is parallel to $\operatorname{Ann}\left(\mathfrak{t}_{j}\right)$. When the restriction of $\phi$ to $F_{j}$ is shifted to $\operatorname{Ann}\left(\mathfrak{t}_{j}\right)=\left(\mathfrak{t} / \mathfrak{t}_{j}\right)^{*}$ it becomes a moment map for the action of $T / T_{j}$. Hence, by the convexity theorem, the image $\phi\left(F_{j}\right)$ is a convex polytope in its own right, though of dimension $d-d_{j}$. In particular, if $T_{j}=T$, then $\phi\left(F_{j}\right)$ is a point.

It is important to note that two different $F_{j}$ can have overlapping, or even identical, images under $\phi$. Tolman [Tol98] introduced the notion of the $X$-ray of $M$ to keep track of this data.

Definition 3.1. The $\mathbf{X}$-ray of a compact Hamiltonian $T$-space as above is the family $\left\{\phi\left(F_{j}\right)\right\}$ of convex polytopes in $\mathfrak{t}^{*}$, indexed by the set $\mathcal{F}$, where $\mathcal{F}$ is partially ordered under inclusion. A wall of the X-ray is a pair $\left(F_{j}, \phi\left(F_{j}\right)\right.$.

More technically, the data of the X-ray are the partial order $\mathcal{F}$ and the map $F_{j} \mapsto \phi\left(F_{j}\right)$, which we will also denote by $\phi$. We will often refer to an X-ray by the pair $(\mathcal{F}, \phi)$. Note that $F_{j} \subset F_{k}$ implies $\phi\left(F_{j}\right) \subset \phi\left(F_{k}\right)$ but not vice versa. We will refer to the walls coming from $T$-fixed point components (which are just single points of $\mathfrak{t}^{*}$ ) as the vertices of the X-ray. We will sometimes abuse notation and refer to $F_{j}$, or $\phi\left(F_{j}\right)$, as a wall.

$\mathrm{X}$-rays have some nice formal properties (which are explored further in [Met97]). Denote the affine span of a set $S$ in $\mathfrak{t}^{*}$ by $\operatorname{Aff}(S)$, and the unique linear subspace parallel to $\operatorname{Aff}(S)$ by $\operatorname{Lin}(S)$.

Proposition 3.2. Given a compact Hamiltonian $T$-space $M$, its $X$-ray map $F_{j} \mapsto$ $\phi\left(F_{j}\right)$ is an order-preserving map from $\mathcal{F}$ to the set of convex polytopes in $\mathbf{t}^{*}$, satisfying:

1. Given $F_{j} \in \mathcal{F}$, and a face $\delta$ of $\phi\left(F_{j}\right)$, there is a unique $F_{k} \leq F_{j}$ such that $\phi\left(F_{k}\right)=\delta$.

2. Given $F_{j} \in \mathcal{F}$, and $F_{k} \geq F_{j}, F_{l} \geq F_{j}$, with $\operatorname{Lin}\left(\phi\left(F_{k}\right)\right)=\operatorname{Lin}\left(\phi\left(F_{l}\right)\right)$, then $F_{k}=F_{l}$.

3. $F_{k}<F_{j} \Longrightarrow \operatorname{dim} \phi\left(F_{k}\right)<\operatorname{dim} \phi\left(F_{j}\right)$.

\footnotetext{
${ }^{3}$ Not all connected components of $M^{T_{j}}$ are of this form; for example a connected component of $M^{T}$ will often be a connected component of $M^{T_{j}}$ as well, but it is not the closure of a stratum corresponding to $T_{j}$.
} 
Proof. Denote the infinitesimal stabilizer of a generic point in $F_{k}$ by $\mathfrak{t}_{k}$. Note that $\operatorname{Lin}\left(\phi\left(F_{k}\right)\right)=\operatorname{Ann}\left(\mathfrak{t}_{k}\right)$ by the definition of a moment map. Hence part 3 above follows from the fact that for any $F_{j} \subsetneq F_{k}, T_{j} \supsetneq T_{k}$, and hence $\mathfrak{t}_{j} \supsetneq \mathfrak{t}_{k}$, since $T_{j}, T_{k}$ are connected. Also, part 2 is clear: the condition $\operatorname{Lin}\left(\phi\left(F_{k}\right)\right)=\operatorname{Lin}\left(\phi\left(F_{l}\right)\right)$ implies that $F_{k}$ and $F_{l}$ are connected components of the fixed point set of the same subtorus. But they both contain $F$, hence they must be equal.

Part 1 is a bit deeper. It comes from the result of Atiyah Ati82 that the inverse image of any point under the moment map is connected. Consider a stratum $F_{j}$. This is a Hamiltonian $T$-space. Given a face $\delta$ of $\phi\left(F_{j}\right)$, let $\mathfrak{h}=\operatorname{Ann}(\operatorname{Lin}(\delta))$, and let $H \subset T$ be the corresponding Lie subgroup. (This exists since the polytope $\phi\left(F_{j}\right)$ is rational Gui94 4.) Consider the moment map of the $H$-action on $F_{j}$; this is just the projection of the moment map by $\pi_{H}: \mathfrak{t}^{*} \rightarrow \mathfrak{h}^{*}=\mathfrak{t}^{*} / \operatorname{Lin}(\delta)$. This moment map has an extremum at $\pi_{H}(\delta)$, which is certainly a critical value. Moreover, any point in $F_{j}$ mapping to this point will necessarily be a critical point. Hence the entire inverse image of $\pi_{H}(\delta)$ under the $H$-moment map, which is the inverse image of $\delta$ under the $T$-moment map, is fixed by the action of $H$. Moreover, this set is connected by Atiyah's result. Hence it is exactly some $F_{k}$, and $H=T_{k}, \phi\left(F_{k}\right)=\delta$, and no other $F_{l} \subset F_{j}$ can map to $\delta$.

Remark. Tolman's X-rays do not satisfy part 3 above, since she deals with a finer stratification of $M$. We should note, however, that much of what we say in the rest of this section applies in a slightly modified form to Tolman's X-rays.

Example. We again consider a complex projective space, as on page 9, but now we take $n=4, d=2$. For a certain choice of the subtorus, the image of the moment polytope will appear as in Figure 3 (This picture is in $\mathbb{R}^{2}$.) The five dots are the vertices (images of the $T$-fixed point components), while the lines are images of strata corresponding to circles in $T$.

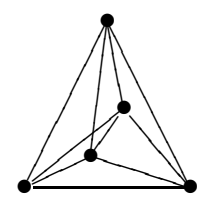

Figure 3. X-ray of $M=\mathbb{C P}^{4}$, with 2-torus action

3.1. Weight data. We also want to record information about the weights of the infinitesimal action of $T$ on the normal bundles to the strata. This data will appear in the wall-crossing formulas for the signature and other invariants. Moreover, the weight data at the $T$-fixed points often completely determine the $\mathrm{X}$-ray (see [Met97]).

Recall from the introduction that the $T$-invariant symplectic structure on $M$ defines a natural isotopy class of compatible, invariant almost complex structures. Hence, given any point $p \in M^{T_{j}}$, the complex weights of the action of $T_{j}$ on $T_{p} M$ are well-defined as vectors in $\mathfrak{t}^{*}$. Given a stratum $F_{j} \subset M^{T_{j}}$, denote the weights of

\footnotetext{
${ }^{4}$ This is another feature of Hamiltonian $T$-spaces that will not be particularly relevant in this paper.
} 
the $T_{j}$ action on $T_{p} M$, for any $p \in M$, by $\alpha_{j}=\left\{\alpha_{j, k} \in \mathfrak{t}_{j}^{*}\right\}$. (Since $F_{j}$ is connected, this is independent of $p$.)

Definition 3.3. The weighted $X$-ray of $M$ is the X-ray together with the assignment to each stratum $F_{j}$ of the weights $\alpha_{j, k} \in \mathfrak{t}_{j}^{*}$.

Note that we consider all of the weights on $\left.T M\right|_{F_{j}}$, not just the weights of the normal bundle $N_{F_{j}}$. Of course the additional weights of $T_{j}$, corresponding to $T F_{j}$, are zero, but we will see below that the bookkeeping works out better if we include these weights as well. Also, $\alpha_{F}$ is not a set of vectors, but a family, as we need to keep track of multiplicities.

We need to describe some of the properties that weighted X-rays of Hamiltonian $T$-spaces have. The most important is a consequence of the equivariant Darboux theorem, which says that the weights determine the X-ray "locally."

We will use the following definition of a local model. In any vector space $A$, given a set of vectors $\alpha=\left\{\alpha_{1}, \ldots, \alpha_{n} \in A\right\}$, denote the positive cone generated by $\alpha$ by

$$
\text { Cone }\left(\alpha_{1}, \alpha_{2}, \ldots \alpha_{n}\right)=\left\{\sum_{k=1}^{n} t_{k} \alpha_{k} \mid t_{k} \geq 0\right\} \text {. }
$$

Call a subset $S=\left\{\alpha_{i_{1}}, \alpha_{i_{2}}, \ldots \alpha_{i_{j}}\right\} \subset \alpha$ linear if no other $\alpha_{i} \in \alpha, \alpha_{i} \notin S$ lies in the linear span of $S$; in other words, if $S=\alpha \cap V$ for some linear subspace $V \subset A$.

Definition 3.4. The local model generated by $\left\{\alpha_{1}, \ldots, \alpha_{n} \in A\right\}$ is the set of cones

$$
\{\text { Cone }(S) \mid S \text { a linear subset of } \alpha\} \text {. }
$$

We will also refer to the intersection of these cones with any neighborhood $U$ of 0 as a local model.

We will also need to consider the natural maps $\pi_{j k}: \mathfrak{t}_{j}^{*} \rightarrow \mathfrak{t}_{k}^{*}$ arising when $F_{j} \subset F_{k}$, and the quotient map $\pi_{j}: \mathfrak{t}^{*} \rightarrow \mathfrak{t}_{j}^{*}$.

Proposition 3.5. The weighted $X$-ray $(\mathcal{F}, \phi, \alpha)$ of a Hamiltonian $T$-space satisfies:

1. for every stratum $F_{j}$, there is a neighborhood $U \subset \mathfrak{t}^{*}$ of $\phi\left(F_{j}\right)$ such that $\left\{\pi_{k}\left(\phi\left(F_{k}\right) \cap U\right) \mid F_{k} \supseteq F_{j}\right\}$ is the local model generated by $\alpha_{j}$;

2. for every $F_{j}, F_{k} \in \mathcal{F}, F_{j} \subseteq F_{k}$, the families of vectors $\pi_{j k}\left(\alpha_{j}\right)$ and $\alpha_{k}$ are equal, up to rearrangement. (I.e. they contain the same vectors with the same multiplicities.)

We will refer to condition 1 as the Darboux condition, and condition 2 as the consistency condition.

Proof. The weights of the action of $T_{k}$ on the tangent bundle can be determined by looking at the tangent space to any point $p \in F_{k}$. Suppose we have $F_{j} \subseteq F_{k}$. Choose the point $p$ to be in $F_{j}$. Then the representation of $T_{j}$ on the tangent space $T_{p}(M)$ must restrict to the representation of $T_{k}$. In terms of weights, this is exactly condition 2 in the proposition.

To show that the Darboux condition holds for an arbitrary wall, first note that it is enough to show that it holds for the fixed points of the whole torus. For, given a stratum $F_{j} \subset M^{T_{j}}$, we can restrict the action to $T_{j}$; the effect on the moment map is to compose with the projection $\pi_{j}: \mathfrak{t}^{*} \rightarrow \mathfrak{t}_{j}^{*}$. Clearly the formation of a local model, and hence the Darboux condition, respects this projection, so $F_{j}$ will 
satisfy the Darboux condition with respect to the $T$ action iff it satisfies it with respect to the $T_{j}$-action.

So assume that $p \in F \subset M^{T}$. Denote the weights of the action of $T$ on $T_{p} M$ by $\alpha=\left\{\alpha_{1}, \ldots \alpha_{n}\right\}$. The Darboux theorem, in the equivariant setting, says ([GS84], p. 251) that we can equivariantly identify a neighborhood of $p$ with $\left(\mathbb{C}^{n}, \omega_{s t d}, \phi_{\alpha}\right)$, where $\mathbb{C}^{n}$ has the action of $T$ given by the weights $\alpha_{k}$, that is, the infinitesimal action of $v \in \mathfrak{t}$ is given by

$$
v \cdot z=\left(i \alpha_{1}(v) z_{1}, \ldots, i \alpha_{n}(v) z_{n}\right),
$$

and

$$
\begin{gathered}
\omega_{s t d}=\frac{i}{2} \sum_{k=1}^{n} d z \wedge d \bar{z}, \\
\phi_{\alpha}(z)=\frac{1}{2} \sum_{k=1}^{n}\left|z_{k}\right|^{2} \alpha_{k} .
\end{gathered}
$$

Hence we only need to determine the relationship of the weights to the images of the fixed point sets of subtori for this linear space.

Given a subspace $W \subset \mathfrak{t}$, let $V=\operatorname{Ann}(W)$. The set of points in $\mathbb{C}^{n}$ infinitesimally fixed by all $w \in W$ is

$$
\left(\mathbb{C}^{n}\right)^{W}=\left\{\left(z_{1}, \ldots, z_{n}\right) \mid \forall k, z_{k} \neq 0 \Longrightarrow \alpha_{k} \in \operatorname{Ann}(W)\right\}
$$

and its moment image is

$$
\begin{aligned}
\phi\left(\left(\mathbb{C}^{n}\right)^{W}\right) & =\left\{\frac{1}{2} \sum_{k=1}^{n}\left|z_{k}\right|^{2} \alpha_{k} \mid z_{k} \neq 0 \Longrightarrow \alpha_{k} \in V\right\} \\
& =\text { Cone }(\alpha \cap V) .
\end{aligned}
$$

Since any $V$ is the annihilator of some $W$, the walls are exactly the sets of the form (3.2), which proves the Darboux condition.

The Darboux condition involves both existence and uniqueness: for every linear subset $S$ of the weights of $F_{j}$, there is a unique wall, comparable to $F_{j}$, that locally looks like the cone on $S$. However, the uniqueness only applies to strata $F_{k} \supseteq F_{j}$; there may be other walls which happen to lie near $F_{j}$ (or even overlap it) but if they are not comparable to $F_{j}$, they need have no particular relation to it.

Note that one consequence of the Darboux condition is that the weights of the $T$-fixed point components lie along the one-dimensional walls of the X-ray. Since for our purposes we will not need to know the length of the weights, it is not always necessary to draw the weights explicitly, since we can read off their directions from the 1-skeleton of the X-ray. However, there are often multiple weights pointing in the same direction, and we will need to be careful to record this multiplicity information.

In the example of $\mathbb{C P}^{4}$ above (Figure 3), each $T$-fixed point is isolated, and hence has four weights attached to it. Clearly there must be exactly one weight lying in the direction of each line emanating from a given vertex. Here there is no ambiguity in the multiplicities, and we can read off all of the information we will need from the figure.

So far we have concentrated on the walls of the X-ray, which come from the various fixed point sets. Now we look at the complement of the walls. Assume 
from now on that $M$ is connected. It is easy to see that the union of the walls (not counting the largest "wall," the entire moment polytope) is the set of singular values of the moment map, and hence the complement is the set of regular values:

$$
\Delta_{\text {reg }}=\Delta \backslash \bigcup_{F \neq M} \phi(F) .
$$

As mentioned in the introduction, this is an open set with a finite number of components, which we call chambers. It is well-known that these chambers are open convex polytopes. (In fact the convexity follows from Propositions 3.2 and [3.5, as we prove in [Met97.)

We will want the following more general definition when we discuss recursive invariants in Section 4. Recall that a stratum $F_{j} \subset M^{T_{j}}$ is an effective Hamiltonian $T / T^{j}$ space. The regular values of the restricted moment map are clearly

$$
\operatorname{Reg}\left(F_{j}\right)=\phi\left(F_{j}\right) \backslash \bigcup_{F_{k}<F_{j}} \phi\left(F_{k}\right),
$$

which is a relatively open set in $\phi\left(F_{j}\right)$, again a finite union of (relatively) open convex polytopes [Met97]. We need to be careful to remember which $F_{j}$ these came from, so call a pair $\left(F_{j}, P\right)$, where $P$ is a component of $\operatorname{Reg}\left(F_{j}\right)$, a subchamber of the X-ray. By abuse of notation we will often refer to $P$ as a subchamber, when it will cause no confusion. Note that the vertices of the X-ray are subchambers: the corresponding torus $T / T$ is trivial, so its Lie algebra is zero-dimensional, and every point is a regular point for the "moment map."

\section{ReCURSIVE INVARIANTS OF X-RAYS}

Given two adjacent chambers in the moment polytope of a Hamiltonian $T$-space, we would like to have a wall-crossing formula that relates the signature of a reduction on one side to that of a reduction on the other side. The simplest analogy with the circle case would be if the difference were expressed in terms of the weights associated to the wall, and the signature of some fixed point component(s). The truth is slightly more complicated. In fact the signatures of the reduced spaces are best understood as part of a larger system, which we will refer to as a recursive invariant.

We know from Proposition 1.1 that topological invariants of reductions, like the signature, are functions only of the set of chambers of the moment polytope. We want to elaborate on this idea slightly. So far, we have only considered the set of regular values $\Delta_{\text {reg }}$ as places where we can reduce. However, every point in the moment polytope is a regular value for some Hamiltonian action. Precisely, let $F_{j} \in \mathcal{F}$ be a stratum corresponding to the subtorus $T_{j}$, and fix a subchamber $P \subset \phi\left(F_{j}\right)$. For any point $q \in P, q$ is a regular value of the moment map for the action of the quotient torus $H_{\sqrt{5}}$, so we can form the regular symplectic reduction

$$
\left(F_{j} / / H_{j}\right)_{q}=\left(\phi^{-1}(q) \cap F_{j}\right) / H_{j}=\left(\phi^{-1}(q) \cap F_{j}\right) / T .
$$

We will denote this by $M_{(q)}^{j}$, and when there is no ambiguity, simply $M_{(q)}$. Note that if $q$ lies in $\Delta_{\text {reg }}$, this is simply the usual symplectic reduction of $M$ at $q$. However, when $q \notin \Delta_{\text {reg }}$, it is to be carefully distinguished from the singular reduction $\phi^{-1}(q) / T$, which is not even an orbifold in general. (In fact it is a symplectic

\footnotetext{
${ }^{5}$ We must technically pick a fixed translation of $\operatorname{Aff}\left(\Delta_{j}\right)$ into $\operatorname{Ann}\left(\mathfrak{t}_{j}\right)$ to say this, but the formula (4.1) doesn't depend on this choice.
} 
stratified space [SL91], and $M_{(q)}^{j}$ is one of the strata.) We must make the usual caveat that this space depends not only on $q \in \mathfrak{t}^{*}$ but also on the fixed point component $F_{j}$. If there are two $F_{j}, F_{k}$ whose moment images both contain $q$, we must specify which fixed point component we are restricting to before taking the reduction.

The recursive formula for the signature, Theorem 4.4, in fact calculates the signature on all of these "subreductions." Clearly, however, the bookkeeping threatens to get a little involved. The weighted X-ray summarizes exactly the data about the Hamiltonian action that is needed.

Fix a Hamiltonian $T$-space $(M, \omega, \phi)$. Denote the weighted X-ray of $M$ by $(\mathcal{F}, \phi, \alpha)$. Let the set of all subchambers $\left(F_{j}, P\right)$ of the X-ray be denoted by $Q_{\mathcal{F}}$. Define a function $S: Q_{\mathcal{F}} \rightarrow \mathbb{Z}$ by

$$
S\left(F_{j}, P\right)=\operatorname{Sign}\left(M_{q}^{j}\right)
$$

for any $q \in P$. We will show that this function satisfies a simple recursive formula. To make things general enough to accommodate the Euler characteristic and the Poincaré polynomial as well, we introduce a general definition. First we need a lemma, concerning how subchambers of an X-ray can meet. Given a wall $(F, \phi(F))$ of an X-ray, call a codimension 1 subwall of $(F, \phi(F))$ a principal subwall.

Lemma 4.1. Let $(\mathcal{F}, \phi, \alpha)$ be the weighted $X$-ray of $(M, \omega, \phi)$. Let $(F, \phi(F))$ be a wall, and let $\left(F, P_{1}\right),\left(F, P_{2}\right)$ be subchambers whose closures intersect in a set which has codimension 1 in $\phi(F)$. Then $P_{12}:=\bar{P}_{1} \cap \bar{P}_{2}$ is a convex polytope. Let $S:=\operatorname{Aff}\left(P_{12}\right)$. Then for every principal subwall $G \subseteq F$ such that $\phi(G) \subset S$ and $\phi(G) \cap P_{12} \neq \emptyset, P_{12}$ lies entirely in one subchamber $(G, R)$ of $G$.

See Figure 4 for a picture of a situation that this lemma rules out, where there are two subchambers in the same wall separating $P_{1}$ and $P_{2}$.

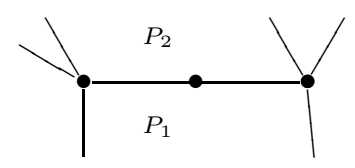

Figure 4. Disallowed meeting of chambers

Proof. Since $\phi(F)$ is an X-ray in its own right, it is no loss of generality to assume that $M$ is connected, that $F=M$, and $P_{1}, P_{2}$ are chambers, intersecting in a principal wall.

Assume that $\bar{P}_{1}$ meets $\bar{P}_{2}$ in two or more (principal) subchambers of $G$. Two of them must be separated by a principal subwall $W$ of $G$. Now $G$ must have weights pointing in the directions of both $P_{1}$ and $P_{2}$. Hence by the consistency condition, $W$ must have weights $\beta_{1}, \beta_{2}$ pointing out of $G$ in the directions of $P_{1}$ and $P_{2}$, respectively. But by the Darboux condition there is a principal subwall of $F$ generated by the codimension 2 wall $W$ and the weight $\beta_{1}$, and likewise for $\beta_{2}$; these walls would break up $P_{1}$ and $P_{2}$, giving a contradiction.

We will be looking at the following situation. Consider a stratum $F \in \mathcal{F}$, and two subchambers $\left(F, P_{1}\right),\left(F, P_{2}\right)$ separated by principal subwalls $\phi\left(G_{1}\right), \ldots, \phi\left(G_{m}\right)$, which have a common affine span $S$. By the lemma, in each wall $\phi\left(G_{i}\right)$ there 
is a unique subchamber $\left(G_{i}, R_{i}\right)$ separating $P_{1}$ and $P_{2}$. Note that since $\phi(G)$ is codimension 1 in $\phi(F), \operatorname{Aff}(\phi(F)) \backslash S$ has two components $C_{1}$ and $C_{2}$, containing $P_{1}$ and $P_{2}$ respectively. Denote the map $\operatorname{Lin}(\phi(F)) \rightarrow \operatorname{Lin}(\phi(F)) / \operatorname{Lin}(S)$ by $\pi$.

Consider the weights of each $G_{i}$ which lie along the wall $F$, i.e.

$$
\alpha_{G_{i}}^{F}=\alpha_{G_{i}} \cap(\operatorname{Lin}(\phi(F)) / \operatorname{Lin}(S)) .
$$

The weights $\alpha_{G_{i}}^{F} \subset \operatorname{Lin}(\phi(F)) / \operatorname{Lin}(S) \cong \mathbb{R}$ divide into three classes: those which are zero, those lying in $\pi\left(C_{1}\right)$, and those lying in $\pi\left(C_{2}\right)$. Let

$$
b_{i}=\#\left\{\alpha_{G_{i}, k} \text { lying in } \pi\left(C_{1}\right)\right\}
$$

and

$$
f_{i}=\#\left\{\alpha_{G_{i}, k} \text { lying in } \pi\left(C_{2}\right)\right\} .
$$

(We think of crossing the wall from $P_{1}$ to $P_{2}$ and $b_{i}, f_{i}$ as the number of weights pointing back and forward, respectively.) In practice, when we determine these numbers from a picture of the X-ray, we can use the consistency condition. We choose any vertex $v_{i}$ belonging to $G_{i}$ and count the weights at $v$ which lie in $\phi(F)$ and which lie on the $C_{1}$ side or the $C_{2}$ side respectively.

We will show that the signature (and the Poincaré polynomial and the Euler characteristic) define invariants of the following form.

Definition 4.2. A recursive invariant $I$ of a weighted X-ray $(\mathcal{F}, \phi, \alpha)$, with values in a ring $R$, consists of two pieces of data:

1. a map $I: Q_{\mathcal{F}} \rightarrow R$, and

2. a function $w_{I}: \mathbb{N} \times \mathbb{N} \rightarrow R$, called the wall-crossing function of $I$.

These must satisfy the following axioms for any wall $F \in \mathcal{F}$.

1. Given two subchambers $\left(F, P_{1}\right),\left(F, P_{2}\right)$ separated by subchambers $\left(G_{i}, R_{i}\right)$, let $b_{i}, f_{i}$ be the number of weights of $G_{i}$ in $F_{i}$ pointing toward $P_{1}$ and toward $P_{2}$ respectively. Then

$$
I\left(P_{2}\right)-I\left(P_{1}\right)=\sum_{i} w_{I}\left(f_{i}, b_{i}\right) I\left(R_{i}\right)
$$

2. Given a subchamber $(F, P)$ adjacent to the boundary of $F$, let $(G, R)$ be the (necessarily unique) subchamber separating $P$ from the exterior of $F$. Let $f$ be the number of weights of $G$ in $F$ pointing into $F$ and $b$ be the number pointing out. Then

$$
I(P)=w_{I}(f, b) I(R) .
$$

Condition 2 is best understood as a special case of condition 1, where one of the "chambers" is the exterior of the wall $F$; in this view, it is understood that we always assign $I=0$ to this "chamber.'

The values of a recursive invariant on a given X-ray are completely determined by the function $w_{I}$ and the values of $I$ on the vertices. The values of $I$ on the subchambers of a given dimension $k$ are determined by its values on the subchambers of dimension $k-1$, along with $w_{I}$. This follows from the fact that we can get to any subchamber of dimension $k$ by starting outside the polytope and crossing a finite number of dimension $k-1$ walls.

\footnotetext{
${ }^{6}$ We avoid actually calling the exterior a chamber because it is neither bounded nor convex; the trade-off is that we have to state condition 2 explicitly.
} 
In fact, this gives an algorithm for calculating $I$ on all of the subchambers of $\mathcal{F}$, starting from the vertices and working our way up. We give two examples in the case of the signature after Theorem 4.4 below.

4.1. Reduction to the circle case. Note that any topological invariant $I$ of symplectic manifolds defines a function on the subchambers of a Hamiltonian Xray by

$$
I\left(F_{j}, P\right):=I\left(M_{(q)}^{j}\right), \text { for any } q \in P .
$$

This invariant will be recursive in general if it is for circle actions:

Theorem 4.3. Let I be a topological invariant of symplectic manifolds. Assume that the X-ray invariant defined by $I$ as in (4.4) is recursive on the class of X-rays coming from Hamiltonian circle actions. Then this invariant is recursive on all Hamiltonian X-rays, with the same wall-crossing function.

Proof. We will show condition 1; condition 2 is very similar. Let $(M, \omega, \phi)$ be a Hamiltonian $T^{d}$-space. Let $\left(F, P_{1}\right),\left(F, P_{2}\right)$ be adjacent subchambers of the X-ray of $M$. We note that everything takes place inside the wall $F$, which is an X-ray in its own right. Hence we can assume that $M$ is connected and effective, $F=M$, and $P_{1}, P_{2}$ are chambers.

By Lemma 4.1 the chambers $P_{1}, P_{2}$ meet in a number of subchambers $\left(G_{j}, R_{j}\right)$ with common affine span $S$. Since the walls $\left(G_{j}, \phi\left(G_{j}\right)\right)$ are principal walls, the $G_{j}$ are fixed point components of circles $T_{j} \subset T$.

Let $a_{i} \in P_{i}$. Choose a $(d-1)$-torus $H \subset T$ such that under the projection $\pi: \mathfrak{t}^{*} \rightarrow \mathfrak{h}^{*}, \pi\left(a_{1}\right)=\pi\left(a_{2}\right)$. Let $L$ be the line $\pi^{-1}\left(\pi\left(a_{i}\right)\right)$. Note that $L$ intersects the walls $\phi\left(G_{j}\right)$ transversely. In Figure 5 we illustrate the nonoverlapping case for simplicity; the projection $\pi$ is in the vertical direction.

Form the symplectic reduction $M_{H}=\phi^{-1}(L)$. This has a residual action of the circle $T / H$, and the reduced spaces of $M_{H}$ by this circle action at $a_{1}$ and $a_{2}$ are exactly the reduced spaces $M_{a_{1}}$ and $M_{a_{2}}$. Polarize $T / H$ so that the positive direction points from $a_{1}$ to $a_{2}$.

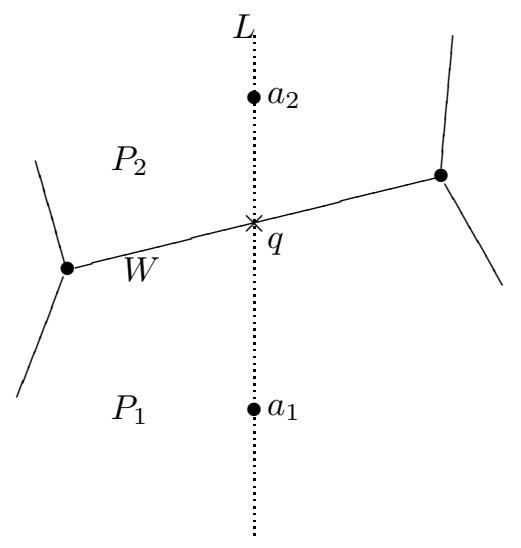

Figure 5. Reduction in stages 
The circle action on $M_{H}$ has one fixed point component lying between $a_{1}$ and $a_{2}$ for each wall $G_{j}$ : these are exactly $\left(G_{j}\right)_{\text {red }}=M_{(q)}^{j}$, where $q=L \cap \phi\left(G_{j}\right)$. The weights of the circle action at these fixed point components are easily calculated. In particular, the positive weights of the circle action at $M_{(q)}^{j}$ are in bijection with the forward weights of $G_{j}$, and the negative weights are bijective with the backward weights.

Hence if the invariant $I$ obeys the wall-crossing formula (4.2) for circle actions, then considering wall-crossing for $M_{H}$ gives

$$
I\left(M_{a_{2}}\right)-I\left(M_{a_{1}}\right)=\sum_{j} w_{I}\left(f_{j}, b_{j}\right) I\left(M_{(q)}^{j}\right) .
$$

But by the definition of the recursive invariant associated to a topological invariant, this amounts to

$$
I\left(P_{2}\right)-I\left(P_{1}\right)=\sum_{j} w_{I}\left(f_{j}, b_{j}\right) I\left(R_{j}\right),
$$

which is the general wall-crossing formula.

Hence any topological invariant which has a formula in terms of weight data like that of the signature - and hence a wall-crossing formula for circle actions - will be a recursive invariant. We show below that this applies not only to the signature, but also to the Poincaré polynomial and the Euler characteristic.

4.2. Application to the signature. The signature is our first example of a recursive invariant. Theorem 4.3 combined with the circle wall-crossing formula (2.8) immediately gives

Theorem 4.4. The signature $S\left(F_{j}, P\right)=\operatorname{Sign}\left(M_{(q)}^{j}\right), q \in P$, is a recursive invariant of Hamiltonian $X$-rays with wall-crossing function

$$
w_{S}(f, b)= \begin{cases}(-1)^{b}=-(-1)^{f} & \text { if } f+b \text { is odd }, \\ 0 & \text { if } f+b \text { is even. }\end{cases}
$$

Note that the vertex function is just the signature of the corresponding fixed point component,

$$
S\left(p_{j}\right)=\operatorname{Sign}\left(F_{j}\right) \text { for } p_{j}=\phi\left(F_{j}\right), F_{j} \subset M^{T} .
$$

This and the wall-crossing function $w_{S}$ determine the signature of all subreductions, and hence all regular reductions, as discussed in the previous section.

4.3. Simple cases. In some cases, this recursive procedure collapses, making the computation much simpler. First, we consider a trivial case.

Suppose $M$ is connected, the action of $T$ is effective, and the dimension of $M$ is twice the rank of the torus. Then the dimension of each regular reduced space is 0 , and since a reduced space is connected, it is just a single point. Hence the signature is always equal to 1 .

Such spaces are called symplectic toric varieties; when they are smooth they are in fact the toric varieties of complex algebraic geometry. They are are characterized by the facts that they have isolated fixed points and that their X-rays have no internal structure, by the work of Delzant Del88. That is, the only walls of the $\mathrm{X}$-ray are the faces of the moment polytope. Further, the moment polytopes of 
toric varieties are simple, meaning that the edges coming out of each vertex form a basis for $\mathfrak{t}^{*}$.

Now consider a general Hamiltonian $T^{d}$-space $M$ and look at its X-ray. Suppose that $M$ is connected and the action is effective, for simplicity. Suppose that the fixed points of $M$ are isolated.

Definition 4.5. Let $\left(F_{j}, \phi\left(F_{j}\right)\right)$ be a wall in the X-ray of $M$, and let $m=$ $\operatorname{dim}\left(F_{j}\right) / 2, r=\operatorname{dim}\left(\phi\left(F_{j}\right)\right)$. The wall $\left(F_{j}, \phi\left(F_{j}\right)\right)$ is Delzant if $m=r$.

Applying Delzant's characterization of symplectic toric varieties to the Hamiltonian $T / T_{j}$-space $F_{j}$ then gives

Proposition 4.6. Let $\left(F_{j}, \phi\left(F_{j}\right)\right)$ be a wall in the X-ray of $M$. The following statements are equivalent:

1. $\left(F_{j}, \phi\left(F_{j}\right)\right)$ is Delzant.

2. $F_{j}$ is a symplectic toric variety with respect to the action of $T / T_{j}$.

3. The only subwalls of $\left(F_{j}, \phi\left(F_{j}\right)\right)$ are its faces.

4. $\left(F_{j}, \phi\left(F_{j}\right)\right)$ has a unique subchamber $P$, and for any $q \in P$, the subreduction $M_{q}^{j}$ is a single point.

If $F_{j}$ is a Delzant wall, by (4) above $\operatorname{Sign}\left(M_{q}^{j}\right)=1$. Hence the recursive procedure terminates early for this particular wall. By (3), we can recognize Delzant walls simply by looking at the X-ray. A Delzant wall $\left(F_{j}, \phi\left(F_{j}\right)\right.$ appears as a wall with "no internal structure," i.e. no subwalls $F_{k} \subseteq F_{j}$ such that $\phi\left(F_{k}\right)$ lies in the interior of $\phi\left(F_{j}\right)$. This can save a great deal of work in applying the recursive procedure for calculating the signature of a reduction $M_{a}$.

The simplest case is described by

Lemma 4.7. Let $M$ be a connected, effective Hamiltonian $T^{d}$-space with isolated fixed points, and let $(\mathcal{F}, \phi, \alpha)$ be its weighted $X$-ray. Then all principal walls of $\mathcal{F}$ are Delzant exactly when for every vertex $p$, any $d$ weights at $p$ are independent.

Proof. First note that the condition that any $d$ weights are independent is equivalent to saying that any $(d-1)$ weights are independent and no other weight lies in their span.

By the Darboux condition, a codimension 1 wall is generated by a set of vectors which span a $(d-1)$-dimensional subspace of $\mathfrak{t}^{*}$. If the wall is Delzant, then it is a simple polytope, hence the weights in that subspace must be independent. So there must be only $(d-1)$ of them. Since no other weight can lie in their span, any $d$ weights will be independent.

Conversely, if any $d$ weights are independent, then the walls are generated by independent sets of $(d-1)$ vectors, hence are Delzant.

In such a case the recursive procedure terminates in codimension 1, giving a simple wall-crossing formula.

Proposition 4.8. Let $M$ be a connected, effective Hamiltonian T-space with isolated fixed points. Assume that all of the principal walls are Delzant. Let $a_{1}, a_{2}$ be regular values of the moment map, separated by walls $F_{1}, \ldots, F_{m}$, and associate $b_{r}, f_{r}$ to each wall as above. Then

$$
\operatorname{Sign}\left(M_{a_{2}}\right)-\operatorname{Sign}\left(M_{a_{1}}\right)=\sum_{r=1}^{m} w_{S}\left(f_{r}, b_{r}\right)
$$




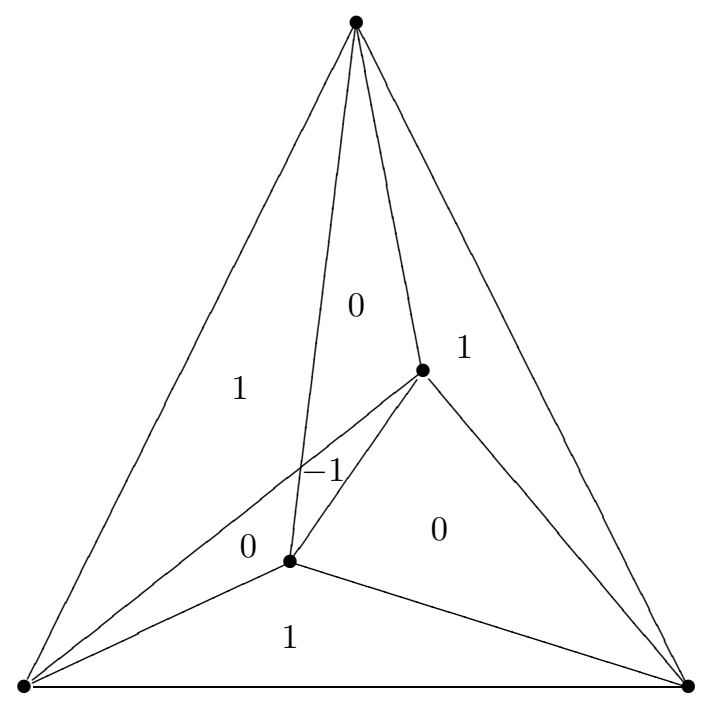

Figure 6. Signatures of reduced spaces of $\mathbb{C P}^{4}$

Example. Consider $\mathbb{C P}^{4}$ as a $T^{2}$-action, as in Section 3 This space has isolated fixed points, and all of the walls are Delzant (in particular, all multiplicities are 1). So the wall-crossing formula gives the signatures shown in Figure 6. For instance, we can calculate the signature in the bottom chamber by crossing the bottom wall from the outside. At either vertex of this wall, there are three weights pointing up, none pointing down. Hence the difference in signature is $W_{S}(3,0)=1$. The other chambers are calculated similarly.

This is not an isolated example. One can show (see [Met97]) that if one starts with any symplectic toric variety and restricts to a generic subtorus $T$, one obtains a Hamiltonian $T$-space whose X-ray has only Delzant walls.

Now we turn to the non-Delzant case, where we do use the recursive version of the formula.

Example. Consider $\mathbb{C P}^{4}$ as a $T^{2}$-space once again, but this time with a non-generic projection, with resulting X-ray given in Figure 7 One wall is now non-Delzant (it is the projection of a 3 -simplex onto a line). We could calculate the signatures in the chambers by crossing the Delzant walls, but we will do it by crossing the non-Delzant wall to show the idea of the recursive formula.

First we need the values on the vertices; but since all of the $T$-fixed points are isolated, $S(p)=S(q)=\cdots=1$.

Next look at the diagonal wall. This is exactly the X-ray considered in the example of Section 2 Hence the signatures of the reductions are

$$
S(A)=S(C)=1, S(B)=0 .
$$

Now we can cross the subchambers $A, B, C$ to find the signatures in the chambers $\alpha, \beta, \gamma$. Crossing from the exterior of the polytope into $\alpha$ through $A$ gives

$$
S(\alpha)=w_{S}(0,1) \cdot S(A)=1,
$$




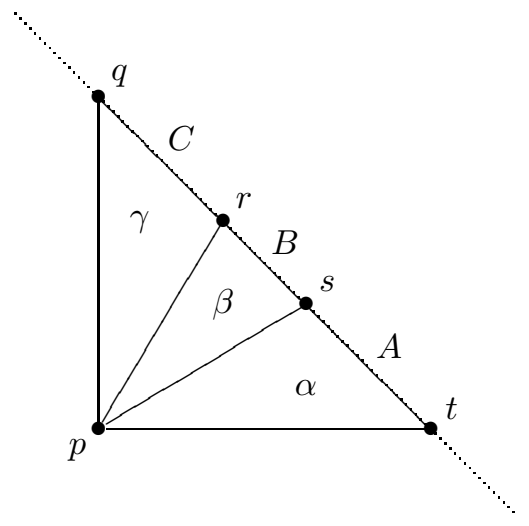

Figure 7. X-ray of $\mathbb{C P}^{4}$ with non-Delzant wall

and similarly $S(\gamma)=1$. Crossing subchamber $B$ gives no change in signature, since $S(B)=0$, so $S(\beta)=0$.

It is easy to see that the results on the chambers accord with what we get by crossing the Delzant walls emanating from the point $p$.

4.4. The Poincaré polynomial and the Euler characteristic. Theorem 4.3 suggests that we look for recursive formulas for other topological invariants. The cobordism approach which yielded Theorem 4.4 will not work for other invariants, because of Theorem 2.9, However, a little Morse theory leads to a wall-crossing formula for the Poincaré polynomial, and hence for the Euler characteristic. We will also note an intriguing connection to the signature formula.

Theorem 4.3 shows that any wall-crossing result can be reduced to the case of a circle action. In that case, we can derive a formula for the change in the Poincaré polynomial as we cross a wall by the following procedure.

Let $(M, \omega, \phi)$ be a Hamiltonian $S^{1}$-space, with fixed point components $F_{1}, \ldots, F_{k}$. Identify $\operatorname{Lie}\left(S^{1}\right)$ with $\mathbb{R}$ as usual. Pick one of the components $F_{r}$, and let $\phi_{r}=$ $\phi\left(F_{r}\right)$. Assume for simplicity that no other fixed point component is mapped under $\phi$ to $\phi_{r}$. Let $a_{1}, a_{2} \in \mathbb{R}$ be regular values of $\phi$ such that $a_{1}<\phi_{r}<a_{2}$, with no other $\phi\left(F_{j}\right)$ lying between $a_{1}$ and $a_{2}$.

We follow the suggestion of Tolman (personal communication) in using the Morse theory of the square of two shifted moment maps, as follows. Kirwan Kir84 showed that $\phi^{2}$ is an equivariantly perfect Morse function 7 Hence so are $\left(\phi-a_{1}\right)^{2}$ and $\left(\phi-a_{2}\right)^{2}$, since the shifted maps are also $S^{1}$ moment maps. Note that the critical sets of $\left(\phi-a_{1}\right)^{2}$ are the fixed points of the action, plus the set $\phi^{-1}\left(a_{1}\right)$; similarly for $\left(\phi-a_{2}\right)^{2}$.

\footnotetext{
${ }^{7}$ Actually, $\phi^{2}$ is not necessarily Morse at the critical value 0 , but 0 is a minimum, and hence the Morse theory still works. See [Kir84] for a detailed discussion.
} 
Calculating the equivariant Poincaré series $\tilde{P}_{M}$ of the manifold $M$ gives 8

$$
\begin{aligned}
\tilde{P}_{M}(t) & =\sum_{F_{j}} P\left(F_{j}\right)(t) \frac{t^{\nu_{j}}}{1-t^{2}}+\tilde{P}\left(\phi^{-1}\left(a_{1}\right)\right)(t) \\
& =\sum_{F_{j}} P\left(F_{j}\right)(t) \frac{t^{\nu_{j}^{\prime}}}{1-t^{2}}+\tilde{P}\left(\phi^{-1}\left(a_{2}\right)\right)(t)
\end{aligned}
$$

where $\nu_{j}$ and $\nu_{j}^{\prime}$ are the indices (the dimensions of the negative normal bundles) of the fixed point set $F_{j}$ with respect to the Morse functions $\left(\phi-a_{1}\right)^{2}$ and $\left(\phi-a_{2}\right)^{2}$ respectively. Note that since $S^{1}$ acts freely on the level sets $\phi^{-1}\left(a_{1}\right)$ and $\phi^{-1}\left(a_{2}\right)$, we have $\tilde{P}\left(\phi^{-1}\left(a_{1}\right)\right)=P\left(M_{a_{1}}\right)$ and similarly for $a_{2}$.

Since only the one fixed point set $F_{r}$ lies between the levels $a_{1}$ and $a_{2}$, we have

$$
\nu_{j}=\nu_{j}^{\prime} \text { for } j \neq r .
$$

Furthermore, let $f$ be the number of positive weights in the normal bundle to $F_{r}$ and $b$ be the number of negative weights. Then $\nu_{r}=2 f$ and $\nu_{r}^{\prime}=2 b$. (Each complex weight gives two real dimensions.) When we equate the two right hand sides in (4.8) above, all of the terms cancel except for

$$
P\left(M_{a_{2}}\right)(t)-P\left(M_{a_{1}}\right)(t)=P\left(F_{r}\right)(t) \frac{t^{2 b}-t^{2 f}}{1-t^{2}} .
$$

It is easy to see that if there are multiple fixed point components $F_{1}, \ldots, F_{m}$ with the same value of the moment map, we get

$$
P\left(M_{a_{2}}\right)(t)-P\left(M_{a_{1}}\right)(t)=\sum_{r=1}^{m} P\left(F_{r}\right)(t) \frac{t^{2 b_{r}}-t^{2 f_{r}}}{1-t^{2}} .
$$

This is the wall-crossing formula for the circle case. Hence we immediately get a wall-crossing formula for a general torus action, by Theorem 4.3 .

Theorem 4.9. The X-ray invariant defined by the Poincaré polynomial,

$$
P\left(F_{j}, R\right)=P\left(M_{(q)}^{j}\right) \in \mathbb{Z}[t], \quad q \in R,
$$

is a recursive invariant of Hamiltonian X-rays with wall-crossing function

$$
\begin{aligned}
w_{P}(b, f) & =\frac{t^{2 b}-t^{2 f}}{1-t^{2}} \\
& =t^{2 f-2}+t^{2 f-4}+\cdots+t^{2 b}(f>b) \\
& =-t^{2 b-2}-t^{2 b-4}+\cdots-t^{2 f}(b>f) .
\end{aligned}
$$

We get a wall-crossing formula for the Euler characteristic by setting $t=-1$ :

Corollary 4.10. The Euler characteristic $\chi$ defines a recursive invariant with wall-crossing function

$$
w_{\chi}(b, f)=b-f
$$

\footnotetext{
${ }^{8}$ We prefer the notation $P_{M}(t)$ for the Poincaré polynomial but we often use $\mathrm{P}(\mathrm{M})(\mathrm{t})$ for easier reading when there are many subscripts.
} 
Example. Consider again the X-ray of the nongeneric 2-torus action on $\mathbb{C P}^{4}$ in Figure[7] Once again we will start with the non-Delzant wall to see how the recursive formula for $P$ works. We evidently have $P(p)=P(q)=\cdots=1$.

Looking at the diagonal wall, we cross $t$ to go from the outside of the wall to subchamber $A$. The wall-crossing formula gives

$$
P(A)=w_{P}(0,3) P(t)=1+t^{2}+t^{4} .
$$

(Note that this is $P\left(\mathbb{C P}^{2}\right)$, as expected from the discussion on p. 9.) Crossing from $A$ into $B$ through $s$ gives

$$
P(B)=P(A)+w_{P}(1,2) P(s)=1+t^{2}+t^{4}+t^{2} .
$$

Crossing from $B$ into $C$ gives

$$
P(C)=P(B)+w_{P}(2,1) P(r)=1+2 t^{2}+t^{4}-t^{2}=1+t^{2}+t^{4}
$$

as we would expect from symmetry.

Now we cross the subchambers $A, B, C$ into the chambers $\alpha, \beta, \gamma$. Crossing from the exterior of the polytope into $\alpha$ through $A$ gives

$$
P(\alpha)=w_{P}(0,1) P(A)=1+t^{2}+t^{4},
$$

and similarly $P(\gamma)=1+t^{2}+t^{4}$. Crossing subchamber $B$ gives $P(\beta)=1+2 t^{2}+t^{4}$.

Note that the wall-crossing functions (4.15) for the Euler characteristic and (4.7) for the signature agree modulo 2 . This accords with the general fact that $\operatorname{Sign}(M) \equiv$ $\chi(M) \bmod 2$ for all compact oriented manifolds (HBJ92]).

It is an interesting fact (see [Met97]) that the signature of a toric variety is determined by its Poincaré polynomial - in fact

$$
\operatorname{Sign}(V)=P_{V}(\sqrt{-1})
$$

for $V$ a toric variety. (This comes from the fact that the Hodge numbers $h^{p, q}$ vanish for $p \neq q$.)

Comparing the wall-crossing formulas for the signature and the Poincaré polynomial shows that as far as the wall-crossing function is concerned, this still holds:

$$
w_{S}(b, f)=w_{P}(b, f)(\sqrt{-1}) .
$$

Since any recursive invariant is determined by its wall-crossing function and its values on vertices, we have the

Theorem 4.11. Let $(M, \omega, \phi)$ be a Hamiltonian T-space. Assume that the fixed point components $F \subset M^{T}$ are toric varieties. Then

$$
\operatorname{Sign}\left(M_{(q)}^{j}\right)=P\left(M_{(q)}^{j}\right)(\sqrt{-1})
$$

for every $q \in \phi\left(F_{j}\right)$, and in particular,

$$
\operatorname{Sign}\left(M_{a}\right)=P_{M_{a}}(\sqrt{-1})
$$

for every $a \in \Delta_{\text {reg }}$.

For example, if $M$ has isolated fixed points, the hypotheses of the theorem are obviously satisfied. 
Here is a simple consequence. Suppose that $N$ is a compact, connected 4dimensional orbifold that can be expressed as a reduced space for a Hamiltonian torus action with isolated fixed points. Then

$$
\begin{gathered}
P_{N}(t)=1+b_{1} t+b_{2} t^{2}+b_{1} t^{3}+t^{4}, \\
\operatorname{Sign}(N)=P_{N}(i)=2-b_{2} .
\end{gathered}
$$

If the diagonalized intersection form of $N$ has $p$ positive entries and $n$ negative ones, we see that

$$
\operatorname{Sign}(N)=p-n=2-b_{2}
$$

but $b_{2}=p+n$, so $p-n=2-p-n$ or

$$
p=1 .
$$

Hence any such 4-manifold has exactly one 2-class with positive self-intersection.

\section{REFERENCES}

[Ati82] M. F. Atiyah. Convexity and commuting Hamiltonians. Bull. London Math. Soc., 14:115, 1982. MR 83e:53037

[CHS57] S. S. Chern, F. Hirzebruch, and J.-P. Serre. On the index of a fibered manifold. Proc. Amer. Math. Soc., 8:587-596, 1957. MR 19:441c

[Del88] Thomas Delzant. Hamiltoniens periodiques et images convexes de l'application moment. Bull. Soc. Math. France, 116(3):315-339, 1988. MR 90b:58069

[DH82] J. J. Duistermaat and G. J. Heckman. On the variation in the cohomology of the symplectic form of the reduced phase space. Inventiones Mathematicae, 69:259-268, 1982. MR 84h:58051a

[Ful93] William Fulton. Introduction to toric varieties. Number 131 in Annals of Mathematics Studies. Princeton University Press, Princeton, NJ, 1993. |MR 94g:14028

[GGK96] Viktor Ginzburg, Victor Guillemin, and Yael Karshon. Cobordism theory and localization formulas for Hamiltonian group actions. Int. Math. Res. Not., (5):221-234, 1996. MR 97d:57046

[GLS96] Victor Guillemin, Eugene Lerman, and Shlomo Sternberg. Symplectic Fibrations and Multiplicity Diagrams. Cambridge University Press, 1996. MR 98d:58074

[GS82] Victor Guillemin and Shlomo Sternberg. Convexity properties of the moment mapping. Inventiones Mathematicae, 67:491-513, 1982. MR 83m:58037

[GS84] Victor Guillemin and Shlomo Sternberg. Symplectic Techniques in Physics. Cambridge University Press, Cambridge-New York, 1984. MR 86f:58054

[GS89] Victor Guillemin and Shlomo Sternberg. Birational equivalence in the symplectic category. Inventiones Mathematicae, 97:485-522, 1989. MR 90f:58060

[Gui94] Victor Guillemin. Moment maps and combinatorial invariants of Hamiltonian $T^{n}$-spaces. Number 122 in Progress in Mathematics. Birkhäuser, Boston, 1994. MR 96e:58064

[HBJ92] Friedrich Hirzebruch, Thomas Berger, and Rainer Jung. Manifolds and Modular Forms. Aspects of Mathematics, E20 Vieweg, 1992. MR 94d:57001

[Hir66] Friedrich Hirzebruch. Topological Methods in Algebraic Geometry. Springer-Verlag, New York, third edition edition, 1966. (English). MR 34:2573

[JK95] Lisa Jeffrey and Frances Kirwan. Localization for nonabelian group actions. Topology, 34(2):291-327, 1995. MR 97g:58057

[Kar98] Yael Karshon. Moment maps and non-compact cobordisms. J. Diff. Geom., (49):183201, 1998. MR 99h:58064

[Kaw91] Katsuo Kawakubo. The Theory of Transformation Groups. Oxford University Press, Oxford, UK, 1991. MR 93g:57044

[Kir84] Frances Kirwan. Cohomology of quotients in symplectic and algebraic geometry. Number 31 in Mathematical Notes. Princeton University Press, Princeton, 1984. MR 86i:58050

[Kir88] Frances Kirwan. An introduction to intersection homology theory. Number 187 in Research Notes in Mathematics. John Wiley \& Sons, New York, 1988. MR 90e:55013 
[LT] Eugene Lerman and Sue Tolman. Intersection cohomology of $S^{1}$ symplectic quotients and small resolutions. math.DG/9810046.

[LT97] Eugene Lerman and Susan Tolman. Hamiltonian torus actions on symplectic orbifolds and toric varieties. Trans. Amer. Math. Soc., (349):4201-4230, 1997. MR 98a:57043]

[Met97] David Metzler. Topological Invariants of Symplectic Quotients. PhD thesis, MIT, 1997. Available at http://math.rice.edu/ metzler/preprints.html.

[MS95] Dusa McDuff and Dietmar Salamon. Introduction to Symplectic Topology. Oxford University Press, New York, 1995. MR 97b:58062

[SL91] Reyer Sjamaar and Eugene Lerman. Stratified symplectic spaces and reduction. Annals of Mathematics, 134(2):375-422, 1991. MR 92g:58036

[Tol98] Sue Tolman. Examples of non-Kähler hamiltonian torus actions. Inventiones Mathematicae, 131(2):299-310, 1998. CMP 98:09

Department of Mathematics, Rice University, Houston, Texas 77005

E-mail address: metzler@math.rice.edu 